\title{
Spatiotemporal Scenario Data-Driven Decision For the Path Planning of Multiple UASs
}

\author{
Chenyuan He, Yan Wan \\ University of Texas at Arlington \\ Arlington, Texas \\ chenyuan.he@mavs.uta.edu,yan.wan@uta.edu
}

\author{
Junfei Xie \\ Texas A\&M University at Corpus Christi \\ Corpus Christi, Texas \\ junfei.xie@tamucc.edu
}

\begin{abstract}
Modern systems operate in spaiotemporally evolving environments, and similar spatiotemporal scenarios are likely to be tied with similar decision solutions. This paper develops a spatiotemporal scenario data-driven decision solution for the path planning of multiple unmanned aircraft systems (UASs) in wind fields. The solution utilities offline operations, online operations and sptaiotemporal scenario data queries to provide an efficient path planning decision for multiple UASs. The solution features the use of similarity between spatiotemporal scenarios to retrieve offline decisions as the initial solution for online fine tuning, which significantly shortens the online decision time. A fast query algorithm that exploits the correlation of spatiotemporal scenarios is utilized in the decision framework to quickly retrieve the best offline decisions. The solution is demonstrated using simulation studies, and can be utilized in other decision applications where spaiotemporal environments play a crucial role in the decision process and the allowed decision time window is short.
\end{abstract}

\section{CCS CONCEPTS}

- Information systems $\rightarrow$ Spatial-temporal systems.

\section{KEYWORDS}

Data driven decision-making, query, spatiotemporal scenario data, UAS Path Planning, UAS traffic management

\section{ACM Reference Format:}

Chenyuan He, Yan Wan and Junfei Xie. 2019. Spatiotemporal Scenario DataDriven Decision For the Path Planning of Multiple UASs. In SCOPE '19: International Science of Smart City Operations and Platforms Engineering, April 15, 2019, Montreal, QC, Canada. ACM, New York, NY, USA, 6 pages. https://doi.org/10.1145/3313237.3313297

\section{INTRODUCTION}

Unmanned aircraft system (UAS) technologies and their applications in a wide range of civilian domains have been rapidly developed in recent years. With the urgent need of safely integrating UASs into the National Airspace System (NAS) [6], the Federal Aviation Administration (FAA) started to investigate the development

Permission to make digital or hard copies of all or part of this work for personal or classroom use is granted without fee provided that copies are not made or distributed for profit or commercial advantage and that copies bear this notice and the full citation on the first page. Copyrights for components of this work owned by others than ACM must be honored. Abstracting with credit is permitted. To copy otherwise, or republish, to post on servers or to redistribute to lists, requires prior specific permission and/or a fee. Request permissions from permissions@acm.org.

SCOPE '19, April 15, 2019, Montreal, QC, Canada

(C) 2019 Association for Computing Machinery.

ACM ISBN 978-1-4503-6703-5/19/04 . \$ $\$ 15.00$

https://doi.org/10.1145/3313237.3313297 of unmanned aircraft system traffic management (UTM) for the safe and efficient low-altitude airspace operations. UTM aims to provide services for airspace design, path planning, dynamic configuration, dynamic geo-fencing, severe weather and wind avoidance, congestion management, terrain avoidance, and separation management $[8,10]$. In this paper, we develop a spatiotemporal scenario datadriven decision solution for the path planning of multiple UASs, as a data-driven solution that addresses the UTM challenges such as scalability, environmental uncertainties, and real-time decisions.

The problem of multi-UAS path planning has been investigated in the literature. Based on different classification criteria, the problem can be classified into geometric and kinodynamic planning, offline and online planning, 2D and 3D planning, and deterministic and probabilistic planning [13]. The solutions to this problem include optimal control approaches such as dynamic programming, operation research approaches such as mixed integer linear programming, and various numerical optimization techniques such as genetic algorithm, $A^{*}$ algorithm, and particle swarm optimization (PSO). Widely used algorithms also include rapidly-exploring random trees, probabilistic roadmaps (PRM), and artificial potential field-based approaches [18]. Of interest, FasTrack [3] employs a planning model and a tracking model to perform safety control within a guaranteed tracking error bound. We note that uncertain spatiotemporal weather plays a crucial role for UAS path planning, which is not considered in many studies. In addition, to deal with the growing number of UASs, scalabiliy becomes an issue. In [11], the impacts of on- and off- board wireless communication sensing to UAS path planning in an uncertain wind field are analyzed. Paper [12] solves a multi-objective shortest path problem under spatiotemporal weather uncertainties, by decomposing the problem into spatial and temporal segments, and uncertain weather impact is captured as a scaling cost in each segment. In this paper, we provide a solution for multiple-UAS path planning that addresses the weather uncertainty and scalability issues, based on a new spatiotemporal scenario data-driven framework.

Broadly, modern systems operate in spaitotemporally evolving environments, and their dynamics are modulated by the environments in a complicated fashion. Such uncertain spatiotemproal environment, if exploited, can improve the efficiency of end-toend decision-making. To enact the environmental data-driven big data analytics, we define a new data type as the spatiotemporal scenario data. A data point of the spatiotemproal scenario data is composed of a sequence of snapshots, each of which is a spatial map. Different from spatial graphical data [4], temporal data [7, 9], and spatiotemporal data [14] that have been widely studied in the literature, the spatiotemporal scenario data, unique for physical networked dynamic system, feature significant correlations across 
the spatial, temporal, and also spatiotemporal dimensions. In our previous studies, we developed algorithms to analyze and process spatiotemporal scenario data [15-17]. In particular, paper [17] used a multi-resolution moving-window to capture spatiotemporal correlations and developed a distance measure to find the similarity among spatiotemporal scenarios for clustering. In [15], an effective similarity search algorithm was developed to quickly retrieve similar spatiotemporal scenarios from stored databases that explores the correlations among the spatiotemproal dimensions. Based on the idea that similar spatiotemporal scenarios generate similar decision solutions, the spaiotemporal scenario data query can be used to quickly generate optimal decisions [16].

This paper develops the spatiotemporal scenario data-driven decision solution in detail, using UTM (and in particular multiple UASs path planning) as the case study. We construct a database for spatiotemporal wind scenarios, tagged with their optimal path planning decisions. The database is constructed offline using the optimal control method. Based on current wind information, a fast query of similar spaitotemporal wind scenario in the database is conducted to retrieve the best offline path planning decision. The decision is then fine tuned online to obtain the optimal path planning decision for the current weather scenario with significantly shortened online computing time. To model uncertain spatiotemporal wind disturbance, we adopt a reduced-order stochastic influence model which captures the wind dynamics at two levels: the network level and the local level $[1,5]$.

The rest of this paper is organized as follows. Section 2 overviews the spatiotemporal scenario data-driven decision framework for multi-UAS path planning. Section 3 introduces the offline path planning operations, including the spatiotemporal influence model, the optimal control-based path planning solution, and the database construction. Section 4 introduces the online path planning operations, including spatiotemporal scenario data distance measure, fast spatiotemporal scenario data query, online tuning of the queried solution, and database expansion. Section 5 shows the simulation studies. Section 6 concludes the paper.

\section{OVERVIEW OF THE SPATIOTEMPORAL SCENARIO DATA-DRIVEN DECISION SOLUTION}

This section describes the spatiotemporal scenario data-driven decision solution for multi-UAS path planning subject to collision avoidance and uncertain wind impact. The framework is developed based on the assumption that similar spatiotemproal wind scenarios share similar path planning solutions, and hence stored solutions can be leveraged to accelerate the online decision-making process.

The structure of the solution framework is shown in Figure 1. The framework is composed of three main blocks: the offline block, the online block, and the database block. The offline block contains three operations. First, spatiotemporal scenario data are obtained from historical environmental data or environmental models. The second operation is to find an optimal planning solution for each scenario. The third operation is to construct a database with the spatiotemporal scenarios and their corresponding optimal solutions. The online block also contains three operations. The first operation is spaiotemporal senario data query, searching for a scenario from

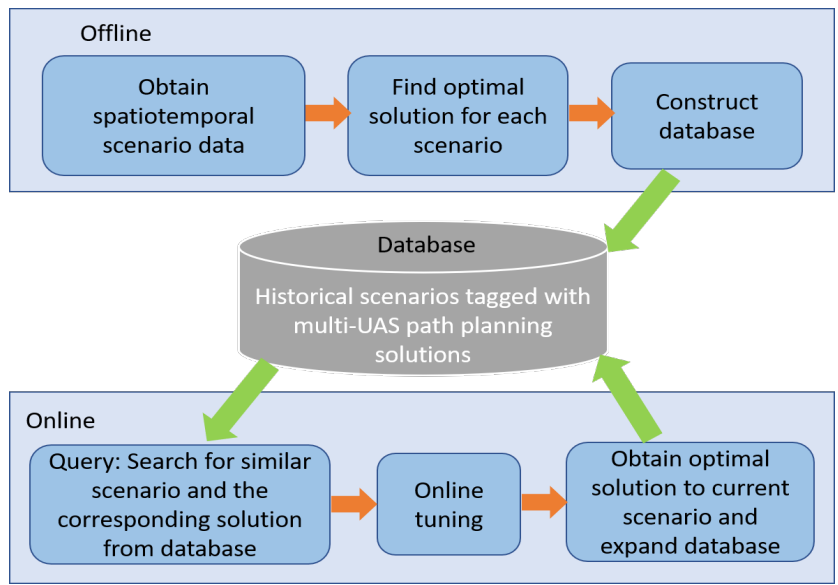

Figure 1: Spatiotemporal Scenario Data-driven Decision Solution.

the database that is similar to the current scenario provided by environmental forecasting tools. The corresponding optimal solution is retrieved. Since the current scenario may not be exactly the same as the retrieved scenario, the retrieved solution needs to be tuned online in the second operation. Once the optimal solution to the current scenario is obtained, it is implemented and meanwhile pushed to the database to expand the solution sets.

We apply the aforementioned spatiotemporal scenario datadriven decision solution to the multi-UAS path planning problem under wind disturbance as a case study. For the offline operations, we use a stochastic influence model to generate spatiotemporal wind scenario data. We then formulate the path planning problem using optimal control. Each UAS has its own dynamics and destination. The objective is to minimize the total trajectory duration subject to wind disturbances and collision avoidance constraints. For the online operations, directly performing exhaustive similarity search is computationally complex and inefficient for spatiotemporal scenario data. We adopt a fast query algorithm which takes advantage of the upper bound and lower bound of the distance measure to accelerate the query process. The optimal path planning solution is obtained through combining the retrieved offline solution with online tuning.

\section{OFFLINE OPERATIONS}

\subsection{Spatiotemporal Wind Scenario Data Generation Using the Influence Model}

We use the influence model, a reduced-order stochastic spatiotemporal network model, to generate the uncertain spatiotemporal wind data $[1,5]$. The model has the following features. First, it simultaneously captures both network- and local- spatiotemporal spreading properties. Spatiotemporal correlations are naturally reflected. In [5], we verified that the model can capture well the spread dynamics. Second, it provides a tractable reduced-order representation of a stochastic network and is computationally efficient.

Consider a wind field of $N$ regions. Each region has its own wind status, which interacts with other regions stochastically. The 


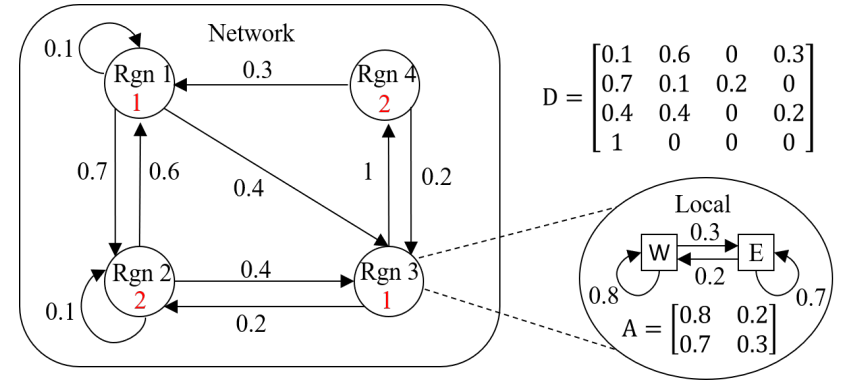

Figure 2: An simple example of a homogeneous influence model of four regions and two wind statuses. 'West' and 'East' are the two statuses of each region.

\begin{tabular}{|c|c|c|}
\hline Wind Velocity & Wind Direction & Status \\
\hline$W_{\min }+\delta_{w} / 2$ & $\Delta_{w} / 2$ & 1 \\
\hline$W_{\min }+\delta_{w} / 2$ & $3 \Delta_{w} / 2$ & 2 \\
\hline$\ldots . .$. & ....... & ....... \\
\hline$W_{\min }+\delta_{w} / 2$ & $2 \pi-\Delta_{w} / 2$ & $1+\left(2 \pi-\Delta_{w}\right) / \Delta_{w}$ \\
\hline$W_{\min }+3 \delta_{w} / 2$ & $\Delta_{w} / 2$ & $2+\left(2 \pi-\Delta_{w}\right) / \Delta_{w}$ \\
\hline$W_{\min }+3 \delta_{w} / 2$ & $3 \Delta_{w} / 2$ & $3+\left(2 \pi-\Delta_{w}\right) / \Delta_{w}$ \\
\hline$\ldots \ldots$ & $\ldots . .$. & $\ldots \ldots$ \\
\hline$W_{\min }+3 \delta_{w} / 2$ & $2 \pi-\Delta_{w} / 2$ & $2+2\left(2 \pi-\Delta_{w}\right) / \Delta_{w}$ \\
\hline$\ldots \ldots$ & $\ldots \ldots$ & $\ldots \ldots$ \\
\hline$W_{\max }-\delta_{w} / 2$ & $\Delta_{w} / 2$ & $\left(2 \pi / \Delta_{w}\left(W_{\max }-W_{\min }-\delta_{w}\right) / \delta_{w}\right)+1$ \\
\hline$W_{\max }-\delta_{w} / 2$ & $3 \Delta_{w} / 2$ & $\left(2 \pi / \Delta_{w}\left(W_{\max }-W_{\min }-\delta_{w}\right) / \delta_{w}\right)+2$ \\
\hline$\ldots \ldots$ & $\ldots \ldots$ & $\ldots \ldots$ \\
\hline$W_{\max }-\delta_{w} / 2$ & $2 \pi-\Delta_{w} / 2$ & $2 \pi / \Delta_{w}\left(1+\left(W_{\max }-W_{\min }-\delta_{w}\right) / \delta_{w}\right)$ \\
\hline
\end{tabular}

Figure 3: The construction of the look-up table that maps velocity and direction into status.

\subsection{Optimal Multi-UAS Path Planning}

We formulate the multi-UAS path planning problem using optimal control [2]. Consider $K$ UASs in a wind field, each of which has its own starting point and destination. Let $X_{i}=\left[x_{i}, y_{i}\right], V_{i}$ and $\theta_{i}$ denote the position, velocity and heading angle of UAS $i$, respectively. The UAS dynamics $\dot{X}_{i}(\tau)=f\left(X_{i}(\tau), \theta_{i}(\tau), \tau\right)$ modulated by wind disturbances can be modeled as:

$$
\left\{\begin{array}{c}
\dot{x}_{i}(\tau)=V_{i}(\tau) \cos \theta_{i}(\tau)+W\left(x_{i}, y_{i}, \tau\right) \cos \phi\left(x_{i}, y_{i}, \tau\right) \\
\dot{y}_{i}(\tau)=V_{i}(\tau) \sin \theta_{i}(\tau)+W\left(x_{i}, y_{i}, \tau\right) \sin \phi\left(x_{i}, y_{i}, \tau\right)
\end{array}\right.
$$

where $W\left(x_{i}, y_{i}, \tau\right)$ and $\phi\left(x_{i}, y_{i}, \tau\right)$ denote the corresponding velocity and direction of wind for the position of UAS $i$ at time $\tau$. We aim to find the optimal solution $\theta_{i}(\tau)$ for all $i \in\{1,2, \cdots, K\}$ to minimize the total trajectory duration for all UASs subject to wind disturbances and collision avoidance constraints. Mathematically, the optimal control problem is formulated as:

$$
\begin{gathered}
\min _{\theta_{i}, i \in\{1,2, \cdots, K\}} J=\sum_{i=1}^{K} \int_{0}^{\tau_{f_{i}}} 1 d \tau \\
\text { Subject to: }\left\{\begin{array}{l}
\dot{X}_{i}(\tau)=f\left(X_{i}(\tau), \theta_{i}(\tau), \tau\right) \\
x_{i}(0)=l x_{i}(0) \\
y_{i}(0)=l y_{i}(0) \\
x_{i}\left(\tau_{f_{i}}\right)=l x_{i}\left(\tau_{f_{i}}\right) \\
y_{i}\left(\tau_{f_{i}}\right)=l y_{i}\left(\tau_{f_{i}}\right) \\
\left|x_{i}(\tau)-x_{j}(\tau)\right|>R \\
\left|y_{i}(\tau)-y_{j}(\tau)\right|>R
\end{array}\right.
\end{gathered}
$$

where $J$ denotes the cost function, $\left[l x_{i}(0), l y_{i}(0)\right]$ and $\left[l x_{i}\left(\tau_{f_{i}}\right), l y_{i}\left(\tau_{f_{i}}\right)\right]$ are the starting point and destination for UAS $i$ respectively, $R$ is the bound of collision distance. We apply time-scaling transformation to the model. Let $t=\frac{\tau}{\tau_{f}}$ and $\frac{d}{d \tau}$ is replaced by $\frac{1}{\tau_{f}} \frac{d}{d t}$. Then we use MATLAB function "fmincon" and Simulink to numerically solve the problem. The Simulink model is shown in Figure 4. The spatiotemporal wind scenario generated by the influence model is stored in MATLAB workspace. We use "WindFunc" to acquire these scenario data and input them to the Simulink model. The optimal control input sequence $\theta_{i}$ for each UAS $i$ is then obtained. 


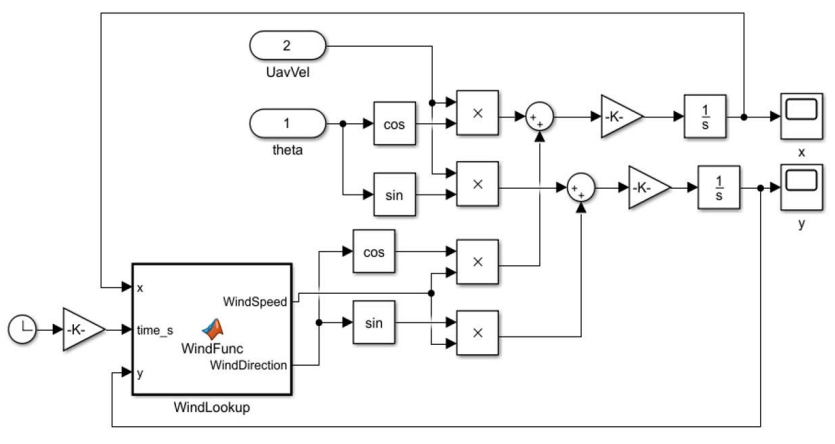

Figure 4: Simulink model for the vehicle dynamics.

\begin{tabular}{|c|c|c|c|c|c|}
\hline \multicolumn{5}{|c|}{ Spatiotemporal Wind Scenarios } & Multiple UAVs Path Planning Strategies \\
\hline s1 & Snapshot 1 & Snapshot 2 & ....... & Snapshot $\mathrm{T}$ & {$\left[\theta_{11}, \theta_{12}, \ldots \ldots \theta_{1 K}\right]$} \\
\hline s2 & Snapshot 1 & Snapshot 2 & ....... & Snapshot T & {$\left[\theta_{21}, \theta_{22}, \ldots \ldots \theta_{2 K}\right]$} \\
\hline s3 & Snapshot 1 & Snapshot 2 & ....... & Snapshot $\mathrm{T}$ & {$\left[\theta_{31}, \theta_{32}, \ldots \ldots \theta_{3 K}\right]$} \\
\hline$\ldots . .$. & & $\ldots . .$. & & & ....... \\
\hline
\end{tabular}

Figure 5: Database structure for the offline solutions.

\subsection{Database Construction}

We construct a database to store the offline spatiotemporal wind scenarios and their corresponding optimal path planning solutions. The structure of the database is shown in Figure 5. The first column stores the tags of scenarios. The second to the $(T+1)$ th columns are spatiotemporal scenario data, each cell of which is a spatial map. The last column stores the corresponding optimal path planning solution for each scenario.

\section{ONLINE OPERATIONS}

\subsection{Distance Measure For Spatiotemporal Scenario Data}

The distance measure we developed in [17] quantifies the similarity between spatiotemopral scenarios generated from physical processes. The distance measure has the following features. First, it captures the spaiotemporal correlation of scenarios using a moving window of multiple resolutions, retaining the spatiotemporal spread patterns of varying shape, size, location and intensity. Second, the distance measure is applicable for not only regular-shaped but also irregular-shaped spatial cells, in the sense that the spaital scanning order of spaiotemporal windows does not matter. Third, the distance measure automatically corrects boundary effects and balances the contributions of all spatial cells and time points. The procedure to calculate the distance matrix among pairs of multiple spatiotemporal scenarios is summarized in Algorithm 1.

Consider two spatiotemporal wind scenarios $s_{i}$ and $s_{j}$ generated according to Section 3.1, each of which is composed of the same number of regions and temporal lengths. Let $B$ denote the set of regions and $b_{n} \in B$ denote a specific region. Likewise, $T$ is the set of time points and $t_{k} \in T$ represents a specific time point. Each region has an intensity at a specific time point, for example, in our case the intensity is the wind status mapped from wind velocity and direction. We denote the wind status of $s_{i}$ at a region $b_{n}$ and time point $t_{k}$ as $I_{i, n, k} \geq 0$.
We use moving windows at spatial and temporal dimensions to simultaneously scan a pair of scenarios and compute their similarity. Let $\phi_{n, w}$ and $\phi_{k, h}$ denote the spatial window and temporal window respectively. $\phi_{n, w}$ is a size- $w$ window centered at the region $b_{n}$ and contains all the regions within $w-1$ hops to $b_{n} . \phi_{k, h}$ is a size- $h$ window starting from the time point $t_{k}$ and contains itself and the subsequent $h-1$ time points. The size ranges of spatial and temporal windows are defined according to the prior-known knowledge of spatiotemporal scenario properties. In general, we choose ' 1 ' to be the minimum window size. Smaller window size indicates finer resolution. The distance between the two scenarios $\mathcal{D}_{i, j, w, h}$ with fixed spatial window size $w$ and temporal window size $h$ is calculated by comparing the aggregated region statuses,

$$
\begin{aligned}
& \mathcal{D}_{i, j, w, h}=\sum_{\phi_{n, w} \in \Phi_{w}} \sum_{\phi_{k, h} \in \Phi_{h}} \frac{1}{\left|\phi_{n, w}\right|\left|\phi_{k, h} \| \Phi_{h}\right|} \\
& \sum_{b_{r} \in \phi_{n, w}} \sum_{t_{l} \in \phi_{k, h}} \frac{I_{i, r, l}}{\lambda_{r, w} \tau_{l, h}}-\sum_{b_{r} \in \phi_{n, w}} \sum_{t_{l} \in \phi_{k, h}} \frac{I_{j, r, l}}{\lambda_{r, w} \tau_{l, h}} \mid,
\end{aligned}
$$

where

$$
\begin{aligned}
\lambda_{n, w} & =\sum_{\phi_{r, w} \in\left\{\phi_{r, w} \mid b_{n} \in \phi_{r, w}\right\}} \frac{1}{\left|\phi_{r, w}\right|} \\
\tau_{k, h} & =\sum_{\phi_{l, h} \in\left\{\phi_{l, h} \mid t_{k} \in \phi_{l, h}\right\}} \frac{|T|}{\left|\phi_{l, h}\right|\left|\Phi_{h}\right|} .
\end{aligned}
$$

$|\cdot|$ denotes the cardinality, $\Phi_{w}$ is the full set of spatial windows of size $w$, and $\Phi_{h}$ is the full set of temporal windows of size $h . \lambda_{r, w}$, a spatial contribution factor, is used to correct the boundary effect of the spatial cells, so that each spatial cell contributes equally to the distance calculation. The temporal contribution factor $\tau_{l, h}$ functions in a similar way.

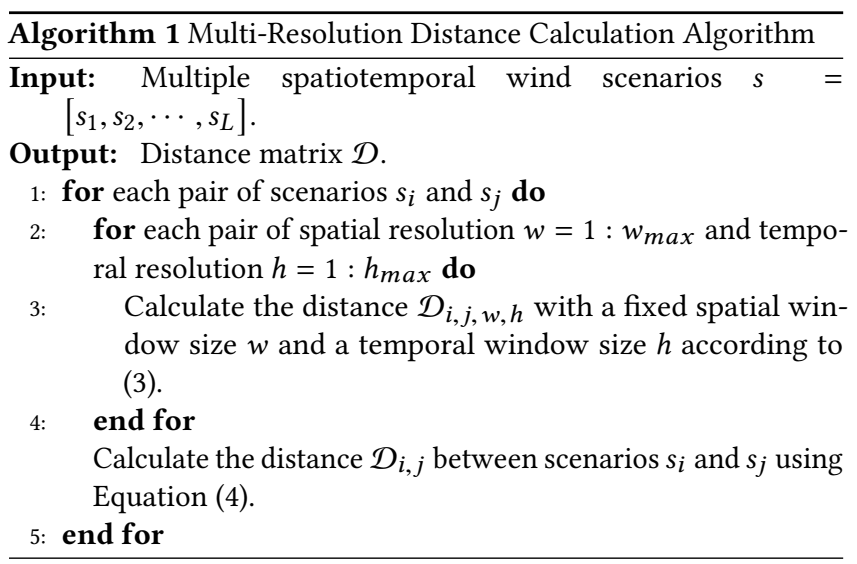

The total distance between $s_{i}$ and $s_{j}$ can be obtained by iterating all the spatial and temporal window sizes in the predefined ranges,

$$
\mathcal{D}_{i, j}=\sum_{h=1}^{h_{\max }} \sum_{w=1}^{w_{\max }} \mathcal{D}_{i, j, w, h} \frac{\sigma_{w} \alpha_{h}}{\sum_{h=1}^{h_{\max }} \sum_{w=1}^{w_{\max }} \sigma_{w} \alpha_{h}},
$$

where $w_{\max }$ and $h_{\max }$ represent the sizes of the maximum spatial window and temporal window respectively, and $\sigma_{w}>0$ and $\alpha_{h}>0$ 
are weighting factors for the spatial and temporal windows, respectively. In general, a larger window size indicates less contribution to the calculation of distance due to its coarse resolution. We select $\sigma_{w}$ and $\alpha_{h}$ to be negative exponential functions as $\sigma_{w}=e^{-\epsilon(w-1)}$ and $\alpha_{h}=e^{-\rho(h-1)}$, where $\epsilon, \rho \geq 0$.

\subsection{Fast Query for Spatiotemporal Scenario Data}

Similar scenarios can be obtained quickly using the distance matrix $\mathcal{D}$. However, computing $\mathcal{D}$ is time-consuming with the increase of scenario number and spatiotemporal window size. The fast query algorithm [15] is used to reduce the computational complexity and accelerate the query process. The basic idea is to trim the searching space after each resolution run by exploiting the bounds of the distance measure. The algorithm is summarized as Algorithm 2.

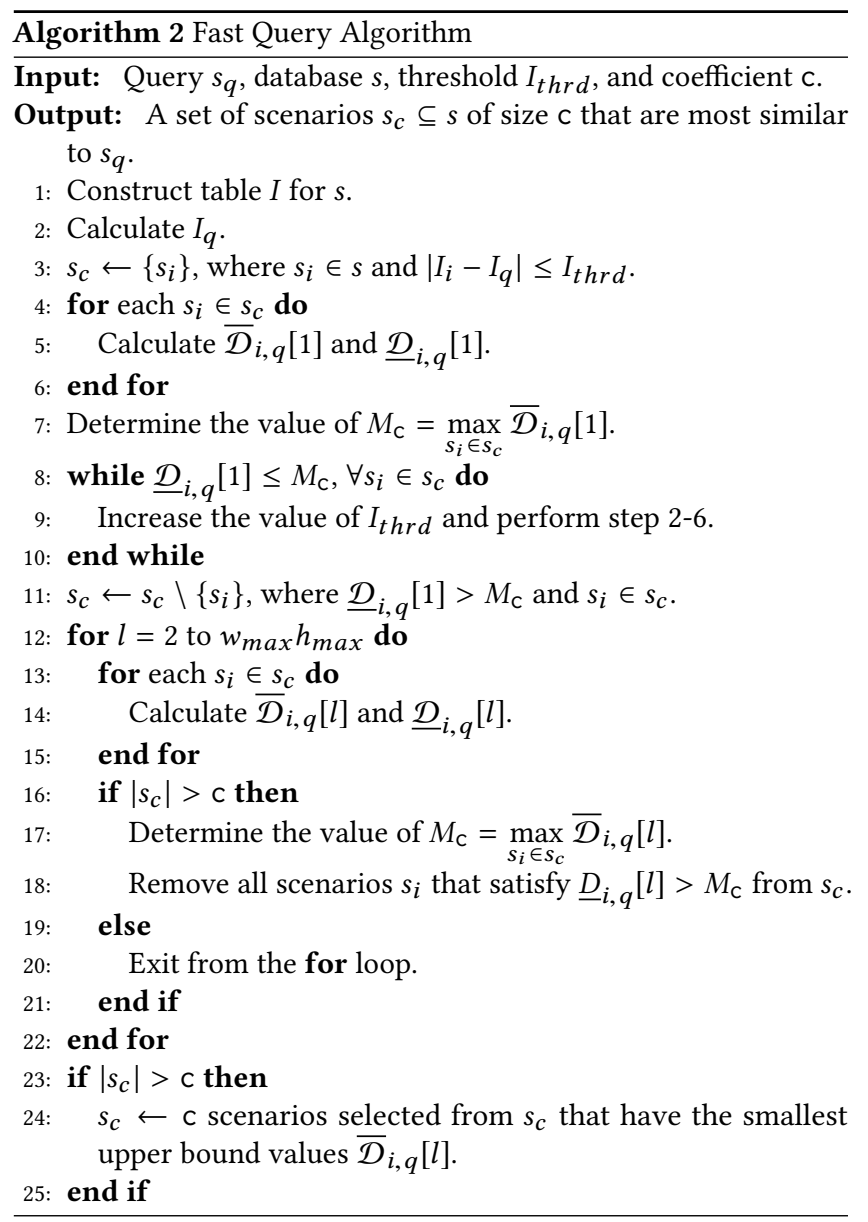

According to (3) and (4), $\mathcal{D}_{i, j, w^{*}, h^{*}} \leq \mathcal{D}_{i, j} \leq \mathcal{D}_{i, j, 1,1}$, where $w^{*}$ and $h^{*}$ are the largest spatial and temporal windows that cover the whole spatial and temporal spaces. Let $I_{i}=\sum_{b_{n} \in B} \sum_{t_{k} \in T i, n, k}$

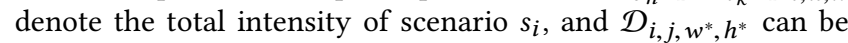
computed as $\mathcal{D}_{i, j, w^{*}, h^{*}}=\frac{\left|I_{i}-I_{j}\right|}{|T|}$. Let $\overline{\mathcal{D}}_{i, j}[l]$ and $\underline{\mathcal{D}}_{i, j}[l]$ denote the upper bound and the lower bound of the distance between $s_{i}$ and $s_{j}$ of spatiotemporal resolution $l$ respectively, where $l \in$

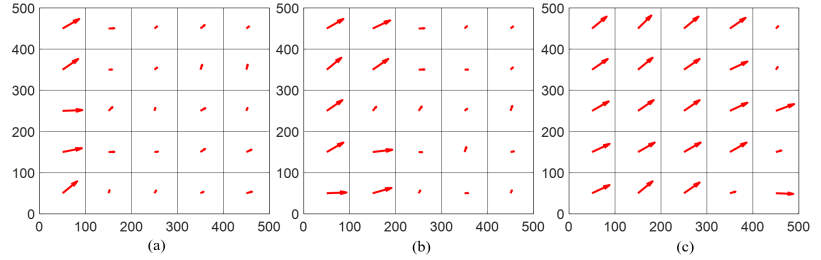

Figure 6: An example of spatiotemporal wind scenario. The arrow at each region denotes the wind velocity and direction. (a) The snapshot at $t=1$. (b) The snapshot at $t=8$. (c) The snapshot at $\mathbf{t}=27$.

$\left\{1,2, \cdots, w_{\max } h_{\max }\right\} \cdot \overline{\mathcal{D}}_{i, j}[l]$ and $\underline{\mathcal{D}}_{i, j}[l]$ can be calculated iteratively using the bounds of finer resolution $l-1$ [15].

Given a query scenario $s_{q}$ and a database $s$, the fast query algorithm includes two main procedures. Procedure 1: Construct a total intensity table $I$. Then an initial candidate set $s_{c}$ can be obtained by applying $\left|I_{i}-I_{q}\right| \leq I_{t h r d}$, where $I_{t h r d}$ is the threshold that limits the number of retrieved scenarios and is determined by statistical analysis of the database. $s_{c}$ is then expanded by comparing the upper and lower bounds of $\mathcal{D}_{i, q}[1]$. Procedure 2: Trim $s_{c}$ based on $\overline{\mathcal{D}}_{i, q}[l]$ and $\underline{\mathcal{D}}_{i, q}[l]$ at each resolution $l$.

\subsection{Online Tuning and Database Expansion}

We adopt the automatic algorithm configuration method for the online tuning operation. The optimal control solution for the most similar scenario retrieved from database is used as the initial solution for the current scenario. The online tuning follows the same optimization procedure that we adopted for the offline solution, with the only change on using the retrieved solution as the initial solution. The database then be expanded by adding the current scenario and its corresponding optimal control solution.

\section{SIMULATION STUDIES}

We demonstrate the spatiotemporal scenario data-driven decision framework for multi-UAS path planning using simulation studies.

We use the influence model to generate 100 spatiotemporal wind disturbance scenarios in a wind field of $5 \times 5$ grids. For each grid, the wind speed ranges from 1 to $10 \mathrm{~m} / \mathrm{s}$ with resolution 1 , and the wind direction ranges from 0 to $2 \pi$ with interval $\frac{1}{36} \pi$. Mathematically, $W_{\text {min }}=1$ and $W_{\max }=10, \delta_{w}=1$ and $\Delta_{w}=\frac{1}{36} \pi$. According to Figure 3 , the total number of statuses is 648 . The temporal length of each scenario is $T=38$. See Figure 6 for an example.

Consider four UASs operating in the wind field, each of which has its own starting point and destination. The velocity of each UAS is $15 \mathrm{~m} / \mathrm{s}$. We follow the offline operations to obtain the optimal path planning solutions and construct the database for the 100 spatiotemporal wind scenarios. For each new spatiotemporal wind scenario, we apply the online operations and obtain its optimal control solution tuned from the retrieved similar scenario.

Figure 7 shows the retrieved and the online turned UAS trajectories for a current wind scenario, together with their optimal control solutions. When there is no wind disturbance and collision avoidance constraints, the four UASs take straight paths from their 


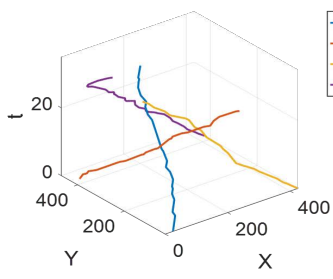

(a)

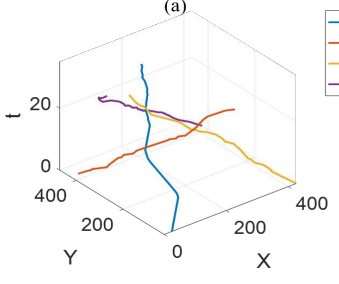

(c)
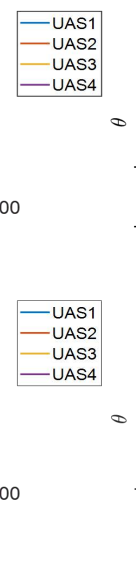

(d)

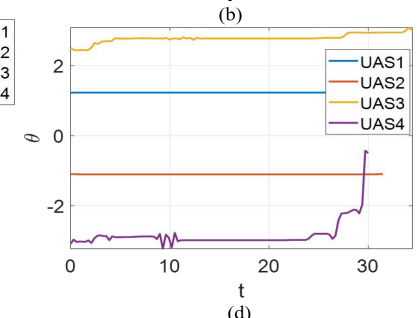

Figure 7: (a) The retrieved 3D UAS trajectories. (b) The retrieved optimal control solution. (c) The 3D UAS trajectories under current wind scenario. (d) The tuned optimal control solution for current scenario. Note that in (a) and (c), to better illustrate the non-collision trajectory, the z-axis denotes time.

starting points to the destinations and the optimal heading angles are constants. To avoid collision under wind, UASs 3 and 4 change their directions during flight, while UASs 1 and 2 remain their heading angle for this spatiotemporal wind scenario. Figures 7 (b) and (d) share similar optimal strategies as we expected, since the two wind scenarios are similar. The difference between the total durations of the two scenarios is also small. The time duration for each UAS under the retrieved scenario is 27, 28.7, 30 and 34.3 and the total duration is 120 . The time duration for each UAS under the current scenario is $27.4,31.5,34.6$ and 30 and the total duration is 123.5 . To compare the online querying and tuning solutions, the direct application of the offline method to the current scenario leads to a total duration of 123.9, with the four UASs' time durations being 28.4, 31.6, 33.6, 30.3, respectively. However, the computational cost is significantly reduced by using the offline, online, and database integrated decision framework.

\section{CONCLUSIONS}

In this paper, a spatiotemporal scenario data-driven decision for multi-UAS path planning is developed. The solution contains offline operations, online operations and a database. The offline operations feature an influence model and an optimal control path planner to generate spatiotemporal scenario data, obtain optimal strategies, and construct the database. The online operations feature a fast query algorithm for spatiotemporal scenario data, online tuning and database expansion. The offline and online operations are integrated to provide computationally efficient decisions based on the similarities of spatiotemporal scenarios. The decision framework can also be utilized in other spatiotemporal environmental applications to meet stringent decision time requirement. In our future work, we will study machine learning-based online tuning algorithm and also consider multi-UAV path planning under stochastic weather scenarios. We will also conduct more numerical experiments with increased database size and the number of UAVs to better evaluate the computational cost benefit.

\section{ACKNOWLEDGMENTS}

We would like to thank NSF grants 1714519 and 1839804 for the support of this work.

\section{REFERENCES}

[1] Chalee Asavathiratham. 2001. The influence model: A tractable representation for the dynamics of networked markov chains. Ph.D. Dissertation. Massachusetts Institute of Technology.

[2] Kevin Bollino and L Ryan Lewis. 2008. Collision-free multi-UAV optimal path planning and cooperative control for tactical applications. In AIAA Guidance, Navigation and Control Conference and Exhibit. Honolulu, Hawaii.

[3] David Fridovich-Keil, Sylvia L Herbert, Jaime F Fisac, Sampada Deglurkar, and Claire J Tomlin. 2018. Planning, fast and slow: A framework for adaptive real-time safe trajectory planning. In 2018 IEEE International Conference on Robotics and Automation (ICRA). Brisbane, Australia.

[4] Jiawei Han, Micheline Kamber, and Anthony KH Tung. 2001. Spatial clustering methods in data mining. Geographic data mining and knowledge discovery (2001), $188-217$.

[5] Chenyuan He and Yan Wan. submitted, 2019. Clustering Stochastic Weather Scenarios using Influence Model-based Distance Measures. In AIAA Aviation Conference. Dallas, TX.

[6] Tao Jiang, Jared Geller, Daiheng Ni, and John Collura. 2016. Unmanned Aircraft System traffic management: concept of operation and system architecture. International journal of transportation science and technology 5, 3 (2016), 123-135.

[7] Konstantinos Kalpakis, Dhiral Gada, and Vasundhara Puttagunta. 2001. Distance measures for effective clustering of ARIMA time-series. In Proceedings 2001 IEEE international conference on data mining. San Jose, California.

[8] Parimal H Kopardekar. 2014. Unmanned aerial system (UAS) traffic management (UTM): Enabling low-altitude airspace and UAS operations. (April 2014). https: //ntrs.nasa.gov/search.jsp?R=20140013436

[9] Carla S Möller-Levet, Frank Klawonn, Kwang-Hyun Cho, and Olaf Wolkenhauer. 2003. Fuzzy clustering of short time-series and unevenly distributed sampling points. In International Symposium on Intelligent Data Analysis. Berlin, Germany.

[10] NASA. 2018. Unmanned Aircraft System (UAS) Traffic Management (UTM). (May 2018). https://utm.arc.nasa.gov/docs/2018-UTM-ConOps-v1.0.pdf

[11] Murilo Augusto Pinheiro, Mushuang Liu, Yan Wan, and Atilla Dogan. 2019. On the Analysis of On-board Sensing and Off-board Sensing through Wireless Communication for UAV Path Planning in Wind Fields. In AIAA Scitech 2019 Forum. San Diego, California.

[12] David Sidoti, Gopi Vinod Avvari, Manisha Mishra, Lingyi Zhang, Bala Kishore Nadella, James E Peak, James A Hansen, and Krishna R Pattipati. 2017. A multiobjective path-planning algorithm with time windows for asset routing in a dynamic weather-impacted environment. IEEE Transactions on Systems, Man, and Cybernetics: Systems 47, 12 (2017), 3256-3271.

[13] Omar Souissi, Rabie Benatitallah, David Duvivier, AbedlHakim Artiba, Nicolas Belanger, and Pierre Feyzeau. 2013. Path planning: A 2013 survey. In Proceedings of 2013 International Conference on Industrial Engineering and Systems Management (IESM). Rabat, Morocco.

[14] Hadi Fanaee Tork. 2012. Spatio-temporal clustering methods classification. In Doctoral Symposium on Informatics Engineering. Porto,Portugal.

[15] Junfei Xie, Akhil Reddy Kothapally, Yan Wan, Chenyuan He, Christine Taylor, and Craig Wanke. 2018, accepted. Similarity Search of Spatiotemporal Scenario Data for Strategic Air Traffic Management. Journal of Aerospace Information Systems (2018, accepted).

[16] Junfei Xie and Yan Wan. 2015. Scalable Multidimensional Uncertainty Evaluation Approach to Strategic Air Traffic Flow Management. In AIAA Modeling and Simulation Technologies Conference. Dallas, TX.

[17] Junfei Xie, Yan Wan, Yi Zhou, Shin-Lai Tien, Erik P Vargo, Christine Taylor, and Craig Wanke. 2015. Distance Measure to Cluster Spatiotemporal Scenarios for Strategic Air Traffic Management. Journal of Aerospace Information Systems 12, 8 (2015), 545-563.

[18] Yijing Zhao, Zheng Zheng, and Yang Liu. 2018. Survey on ComputationalIntelligence-Based UAV Path Planning. Knowledge-Based Systems 158 (2018), $54-64$. 